\title{
pyMolDyn: Identification, structure, and properties of cavities in condensed matter and molecules
}

\author{
Ingo Heimbach ${ }^{\ddagger}$, Florian Rhiem ${ }^{\ddagger}$, Fabian Beule ${ }^{\ddagger}$, David Knodt ${ }^{\ddagger}$, Josef Heinen ${ }^{\ddagger}$, Robert O. Jones ${ }^{\ddagger}$
}

\begin{abstract}
MolDyn is an interactive viewer of atomic systems defined in a unit cell and is particularly useful for crystalline and amorphous materials. It identifies and visualizes cavities (vacancies, voids) in simulation cells corresponding to all seven 3D Bravais lattices, makes no assumptions about cavity shapes, allows for atoms of different size, and locates the cavity centers (the centers of the largest spheres not including an atom center). We define three types of cavity and develop a method based on the split and merge algorithm to calculate all three. The visualization of the cavities uses the marching cubes algorithm. The program allows one to calculate and export pair distribution functions (between atoms and/or cavities), as well as bonding and dihedral angles, cavity volumes and surface areas, and measures of cavity shapes, including asphericity, acylindricity, and relative shape anisotropy. The open source Python program is based on GR framework and GR3 routines and can be used to generate high resolution graphics and videos.
\end{abstract}

Index Terms-Cavity shape, volume, and surface area; Python; marching cubes; split and merge

\section{Introduction}

The properties of many materials are influenced significantly or even dominated by the presence of empty regions, referred to as cavities, vacancies, or voids. In phase change materials, for example, they play an essential role in the rapid and reversible transformation between amorphous and crystalline regions of chalcogenide semiconductors [AJ07], [AJ08], [AJ12]. In soft matter, such as polymers, cavities can lead to structural failure and are often crucial for diffusion of small molecules. Voids caused by radiation (neutrons, x-rays) can lead to dramatic changes in the strength of materials. It is essential to provide efficient algorithms and programs to visualize cavities in the course of computer simulations. We describe here methods developed in the context of phase change materials, where the empty regions are rarely larger than a few atomic volumes, and the term "vacancy" is also in common use [LE11b]. The approach will be useful in other contexts. The present manuscript is an extended and corrected version of [Hetal17].

Geometrical algorithms to identify cavities have a long history in the discussion of disordered materials. Bernal [Be64] discussed liquid structures in terms of space-filling polyhedra and noted that "holes" or "pseudonuclei" would occur in general. Finney

* Corresponding author: i.heimbach@fz-juelich.de

† PGI-1 and PGI/JCNS-TA, Forschungszentrum Jülich, D-52425 Jülich, Germany

Copyright $@ 2017$ Ingo Heimbach et al. This is an open-access article distributed under the terms of the Creative Commons Attribution License, which permits unrestricted use, distribution, and reproduction in any medium, provided the original author and source are credited.
[Fi70] extended this analysis by using the Dirichlet [Di50] or Voronoi [Vo08] construction, where space is divided into regions bounded by planes that bisect interatomic vectors perpendicularly. This construction for a crystalline solid leads to the well-known Wigner-Seitz cell. The polyhedron associated with an atom is the smallest surrounding the atom in question, and its structural features (volume, number of vertexes, etc.) can be used for identification and characterization. A small Voronoi polyhedron indicates an interstitial defect, and a local assembly of large polyhedra could imply the existence of a cavity. This approach has been used to analyze defect structures in simulations of radiation damage [CL85] and the motion of vacancies in colloids [LAC13], although the coordination number (the number of faces of the Voronoi cell) is not necessarily a convenient measure of their positions [LAC13]. Similar techniques have been applied to the distinction between solute and solvent in a liquid, such as hydrated peptide molecules [Vetal11].

Delaunay triangulation [De34], a division of space closely related to the Dirichlet-Voronoi analysis, has been used to identify the "unoccupied space" [AMS92] or "cavities" [VBM15] in polymer systems and to analyze their connectivity, and it has been used to analyze the normal modes in a molecular dynamics simulation of a glass [LMNS00]. Efficient programs are available for performing Voronoi analyses (see, for example, Ref. [Ry09]) and its extension to Voronoi $S$-surfaces, which are appropriate for systems with atoms of different sizes [MVLG06], [VNP]. Ref. [MVLG06] contains many references to physical applications of Dirichlet-Voronoi-Delaunay analyses. The present work and the above approaches focus on the geometrical arrangement resulting from a simulation, rather than determining other physical quantities such as local atomic pressures [LN88].

In the following section, we define essential terms and describe the analysis, based on the "split and merge" [HP76] and "marching cubes" [LC87], [NY06] algorithms, that we have used to study three definitions of cavities:

- Regions (sometimes referred to as "domains") where each point is outside spheres centered on the atoms. The radii of the spheres are generally element-dependent, but an equal cutoff for all elements $(2.5 \AA)$ was chosen in a study of $\mathrm{Ge} / \mathrm{Sb} / \mathrm{Te}$ phase change materials [LE11b].

- $\quad$ "Center-based" cavities resulting from a Dirichlet-Voronoi construction using the atomic positions and the cavity centers.

- $\quad$ "Surface-based" cavities [AJ07], where the boundaries are determined by Dirichlet-Voronoi constructions from each 
point on the domain surface to neighboring atoms, have been used in numerous studies of phase change materials [AJ12], [CBP10], [KAJ14].

The code, the ways to access it, and the online documentation are described below, and an application demonstrates some of its features.

\section{Definitions and Algorithms}

Essential input for a calculation of cavities and their properties is the location of the atoms, which is often provided as a list of coordinates and atom types within an appropriate unit cell. However, the definition of a cavity is not unique and is a prerequisite for any study. Calculation of pair distribution functions involving cavities (with atoms and with other cavities) means that we must also associate appropriate coordinates with the center of each cavity. We now define cavities and describe how we calculate their centers.

\section{Cavity domains and cavity centers}

The first step is the discretization of the simulation cell by creating a cuboid grid containing the cell bounding box and a surrounding layer, which enables periodic boundary condition to be implemented effectively. The resolution $d_{\max }$ refers to the number of points along the longest edge, and two units are added at each end of each cell edge. Each grid point outside the cell has one equivalent point inside. If there are more than one equivalent inside points, we choose the one closest to the origin or-if this is still ambiguous - search for the smallest component in the order $x, y, z$. Outside points are labeled with the index of the translation vector pointing to the equivalent inside point. This step depends only on the cell shape and the resolution of the discrete grid, and the results can be cached and used for other data files.

As shown in Fig. 1(a), we now construct spheres centered on each atom with radii specified for each atom type (element). In earlier work on alloys of Ge/Sb/Te [AJ07], [AJ12] and $\mathrm{Ag} / \mathrm{In} / \mathrm{Sb} / \mathrm{Te}$ [Metal11], the radius was chosen to be the same $(2.8 \AA)$ for all elements $\left[r_{C}\right.$ in Fig. 1(a)]. Points outside the simulation cell are replaced by equivalent points inside. All points outside these spheres form "cavity domains" [yellow in Fig. 1(a)], and the "cavity center" [X in the $2 \mathrm{D}$ scheme $1(\mathrm{~b})]$ is the center of the largest sphere that does not overlap a neighboring atom. It is possible, for example in unusually long, thin cavities, that more than one point satisfy this condition approximately equally well, so that the center can switch between them as a simulation proceeds.

Some structures are unusually sensitive to the choice of cutoff radius $r_{C}$ and/or resolution, particularly when the cavity domains are very small, and it is essential to carry out detailed tests before performing production runs. The program provides a warning when one or more cavity domains consist of a single cell of the discretization grid. The calculation should be repeated with a higher resolution to increase the number of numerically stable cavity domains.

\section{Domains and center-based cavities}

A knowledge of the positions of the atoms and the cavity center enables us to perform a Dirichlet-Voronoi construction (see above) leading to the cavities shown as red in Fig. 1(b). Overlapping cavities from different domains are merged to form "multicavities",

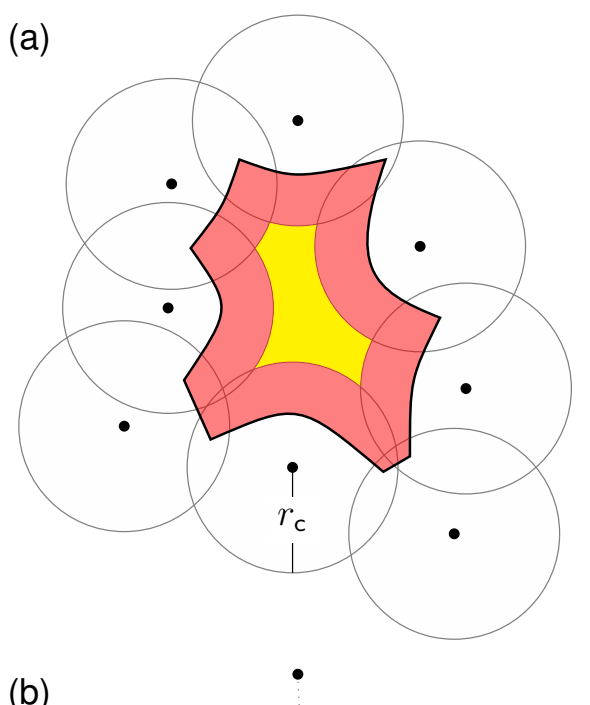

(b)

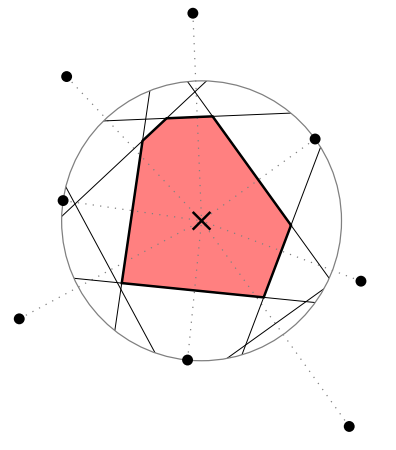

Fig. 1: Construction for a 2D geometry of (a) "cavity domain" (yellow, cutoff radius $r_{\mathrm{C}}$ ) and "surface-based cavity" (red), (b) cavity center (X) and "center-based" cavity (red).

and the volumes and surface areas of cavities and cavity domains are determined as follows.

Points in domains are grouped together by applying the split and merge algorithm [HP76], which consists of three main steps for periodic cells. First, the discrete grid is split recursively into subgrids until they contain either atoms or domain points. Subgrids containing atom points are not needed to determine the domains and are ignored. During the split phase the direct neighbors of each subgrid are recorded, and neighboring subgrid points are then merged to form the cavity domains. As noted above, these domains can be identified as cavities by choosing an appropriate cutoff radius [LE11b].

Center-based cavities comprise points that are closer to domain centers than to any atom, and their construction requires points inside atomic spheres for which there is an atom no farther away than the largest atomic sphere radius. The grid is split into cubes with sides of at least this length, for which only atoms and surface or center points inside neighboring cubes are relevant. If a point is closer to the center of cavity domain $i$ than to the nearest atom, it is marked as part of cavity $i$. In the case of multicavities, intersections are detected by checking the neighboring points of the surface of a cavity. If two such points belong to different cavities, the two cavities are parts of a common multicavity.

The surface of each domain, cavity, or multicavity is important for calculating the surface area and for visualization, and it is determined by applying a variation of the marching cubes algorithm [LC87], [NY06] to new grids based on those derived above. Each grid contains the bounding box of a cavity domain or 
multicavity, and each point in the grid is assigned the number of neighboring points inside the domain or cavity. The algorithm then constructs a surface containing all points with a non-zero count. Neighboring grid points are grouped together into disjoint cubes, and points with a count of 1 are found by interpolation along the edges and connected to form triangles. The set of all such triangles is the surface of a domain or cavity.

\section{Surface-based cavities}

The surface-based cavity [red in Fig. 1(a)] can be determined as for center-based cavities, except that the Dirichlet-Voronoi construction is performed from each point of the domain surface to the neighboring atoms.

\section{Analysis of structure and cavities}

A range of quantities can be calculated for the atomic structure (including bond and dihedral angles) and for each of the above definitions of cavity. In addition to the volume $V_{\mathrm{C}}$, surface area, and location of the center, we calculate the characteristic radius $r_{\text {char }}=\left(3 V_{\mathrm{C}} / 4 \pi\right)^{1 / 3}$, which is the radius of a spherical cavity with volume $V_{\mathrm{C}}$. We also evaluate and export pair distribution functions (PDF) between all atom types and/or cavity centers. Continuous representations can be generated using Gaussian, Epanechnikov [Ep69], compact, triangular, box, right box, and left box window functions. The corresponding kernels are listed in the online documentation, and the default bandwidth $\sigma$ is 0.4 in all cases. Following earlier work [AMS92], [VBM15], [TS85], we calculate the volume-weighted gyration tensor $\mathbf{R}$, which describes the second moment of the coordinates $(x, y, z)$ of points inside a cavity

$$
\mathbf{R}=\frac{1}{V_{\mathrm{C}}}\left(\begin{array}{lll}
\overline{x x} & \overline{x y} & \overline{x z} \\
\overline{y x} & \overline{y y} & \overline{y z} \\
\overline{z x} & \overline{z y} & \overline{z z}
\end{array}\right) .
$$

Here $\overline{x x}=\sum_{j}^{n_{c}} v_{j} x_{j} x_{j}, \overline{x y}=\sum_{j}^{n_{c}} v_{j} x_{j} y_{j}, \ldots, v_{j}$ is the volume of cell $j$, and $n_{c}$ is the number of cells in cavity C. $\left(x_{j}, y_{j}, z_{j}\right)$ are the Cartesian coordinates of the center of cell $j$ relative to the centroid or center of gyration of the cavity, which differs in general from the center defined above. Measures of the size and shape of individual cavities are the squared radius of gyration $R_{g}^{2}$, the asphericity $\eta$, the acylindricity $c$, and the relative shape anisotropy $\kappa^{2}$. These are defined as

$$
\begin{aligned}
R_{g}^{2} & =\lambda_{1}+\lambda_{2}+\lambda_{3} \\
\eta & =\left(\lambda_{1}-0.5\left(\lambda_{2}+\lambda_{3}\right)\right) /\left(\lambda_{1}+\lambda_{2}+\lambda_{3}\right) \\
c & =\left(\lambda_{2}-\lambda_{3}\right) /\left(\lambda_{1}+\lambda_{2}+\lambda_{3}\right) \\
\kappa^{2} & =\left(\eta^{2}+0.75 c^{2}\right) / R_{g}^{4},
\end{aligned}
$$

where $\lambda_{1}, \lambda_{2}$, and $\lambda_{3}$ are the ordered eigenvalues of $\mathbf{R}\left(\lambda_{1} \geq \lambda_{2} \geq\right.$ $\left.\lambda_{3}\right)$.

These quantities provide compact information about the symmetry and overall shape of a cavity and have been useful in the context of diffusants in polymers [AMS92]. The asphericity is always non-negative and is zero only when the cavity is symmetric with respect to the three coordinate axes, e.g. for a spherically symmetric or a cubic cavity. The acylindricity is zero when the cavity is symmetric with respect to two coordinate axes, e.g., for a cylinder. The relative shape anisotropy is bounded by zero (spherical symmetry) and unity (all points collinear). The calculation of these shape parameters requires particular care (and more computer time) when cavities cross the boundaries of the unit cell, and the default is not to calculate these parameters. The parameters are also not calculated for (infinite) cavities that span the simulation cell, and a warning is issued in this case.

\section{Description of the Code}

The program pyMolDyn is written in Python (2.7.13), uses the graphical user interface Qt 5.8.0, the Python module PyQt5 (5.8.2), and the GR Framework and GR3 packages (0.24.0) [HRH15] for 2D- and 3D-graphics, respectively. It has been tested with NumPy (1.12.1). Numerically intensive sections are written in C, compiled using Apple Clang 8.1.0 (macOS) or gec 4.2.1 (Linux) and embedded using ctypes and extension modules. A ready-to-use bundle for OS X (Mavericks, Yosemite, El Capitan) and macOS Sierra is provided at:

http://pgi-jcns.fz-juelich.de/pub/downloads/software/pyMolDyn. dmg

with installation scripts and a package repository for Linux [Debian 8 (Jessie), Ubuntu 16.04 LTS (Xenial Xerus), Centos 7.2, Fedora 25, and OpenSUSE Leap 42.2] at:

https://pgi-jcns.fz-juelich.de/portal/pages/pymoldyn-main.html Documentation is available in the same directory under pymoldyndoc.html, with links to the graphical user and command line interfaces. The source code is available via the public git repository: http://github.com/sciapp/pyMolDyn.

The program supports unit cells of all seven 3D Bravais lattices: triclinic (TRI), monoclinic (MON), orthorhombic (ORT), tetragonal (TET), rhombohedral (RHO), hexagonal (HEX), and cubic (CUB). These cells and the parameters required for their definition are shown in Fig. 2. The bond length cutoffs in all visualizations are $15 \%$ longer than the sum of the covalent radii of the elements [OB], [Cetal08]. The default colors for the elements are those used in Jmol/JSmol [Jmol] and other programs ("CPK", Corey-Pauling-Koltun) [SF].

Each frame to be analyzed requires input in the . xyz-form, which can be read by Jmol/JSmol and other packages. The first lines of an . xyz file are typically:

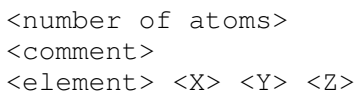

where element is the symbol for the element in question, e.g. $\mathrm{SB}$ or $\mathrm{TE}$, and $\langle\mathrm{X}\rangle,\langle\mathrm{Y}\rangle$, and $\langle\mathrm{Z}\rangle$ are the Cartesian coordinates of the first atom. For each atom there is an input line with its coordinates. In pyMolDyn, the second (usually comment) line provides the necessary information concerning the Bravais lattice and its parameters. In the case of a hexagonal lattice with $a=$ 17.68942 and $c=22.61158$ (in $\AA$ ), for example, we write:

HEX $17.68943 \quad 22.61158$

Additional comments on the first line (after the number of atoms and a space) or the second line are ignored, and the space may be used to store additional information.

The organization of the program and the workflow in practice are clarified in the video linked after the title.

\section{Application}

The use of pyMolDyn is described in detail in the online documentation (see links above). To illustrate its usage, we take the attached input file AgGeS-BOX.xyz, which shows a result of a 500-atom simulation of an amorphous alloy of $\mathrm{Ag}, \mathrm{Ge}$, and $\mathrm{S}$ 


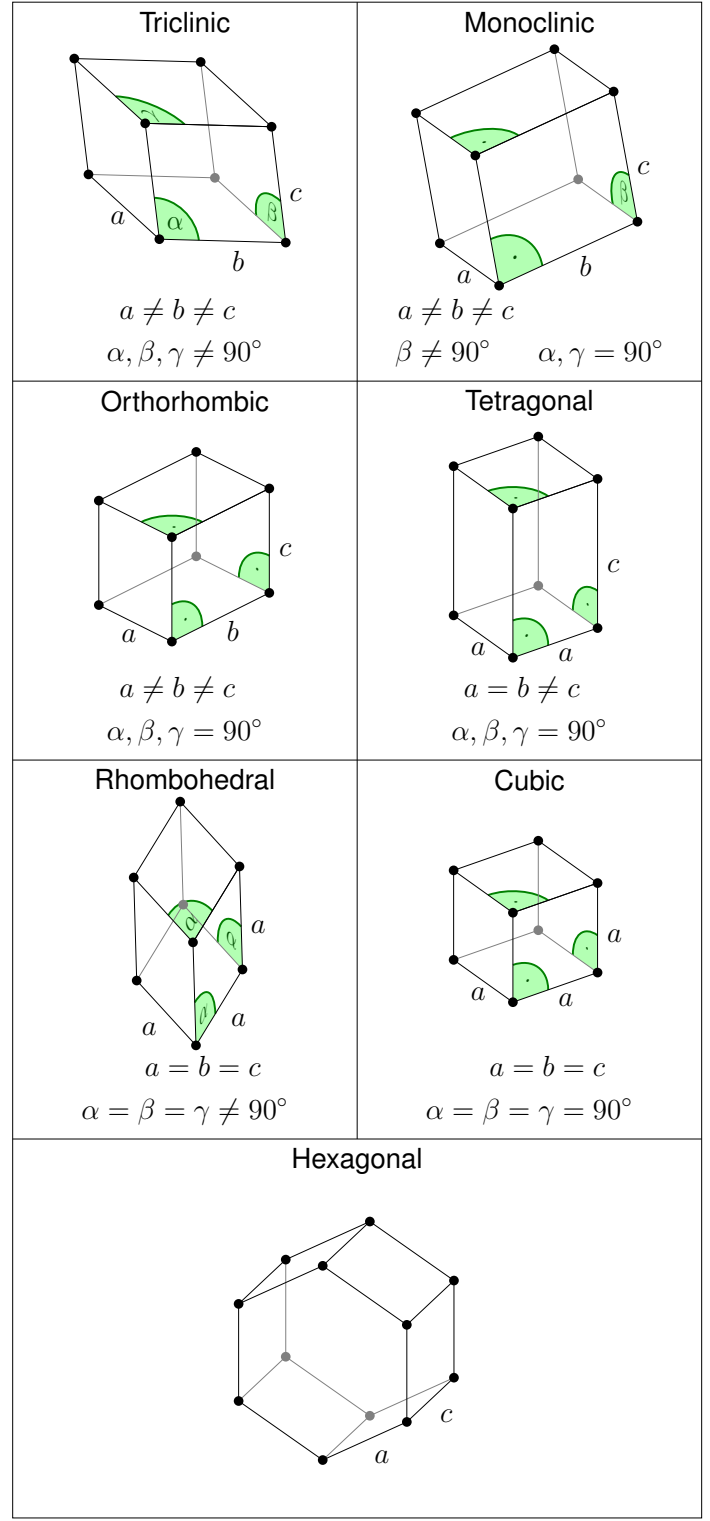

Fig. 2: The unit cells of the seven 3D Bravais lattices, together with the parameters that define them.

$\left(\mathrm{Ag}_{100} \mathrm{Ge}_{168} \mathrm{~S}_{232}\right)$ in a cubic box of size $21.799 \AA$ [Aetal15]. The first three lines of the input are then:

500

CUB 21.799

AG $-7.738 \ldots$

At this point, clicking "pyMolDyn/Preferences" (OS X, macOS) or "File/Settings" (Linux) allows changes to be made to the default colors for background (black), bounding box (white), bonds (grey), domains (green), and center-based (brown) and surfacebased cavities (blue), as well as the cutoff value $r_{\mathrm{C}}$ for calculating surface-based cavities. The default is $2.8 \AA$ for all atoms (we use $2.5 \AA$ in the present application because of the relatively small sulfur atoms), but distinct cutoff radii may be chosen for each element. To guide this choice, the covalent radii for the elements present are provided when setting up the calculation. The resolution can be set by the user and is 384 in the present application. The program is started with the command:

pymoldyn
The choice of file can be made after clicking "Open", after which "Calculate" leads to the window shown in Fig. 3.

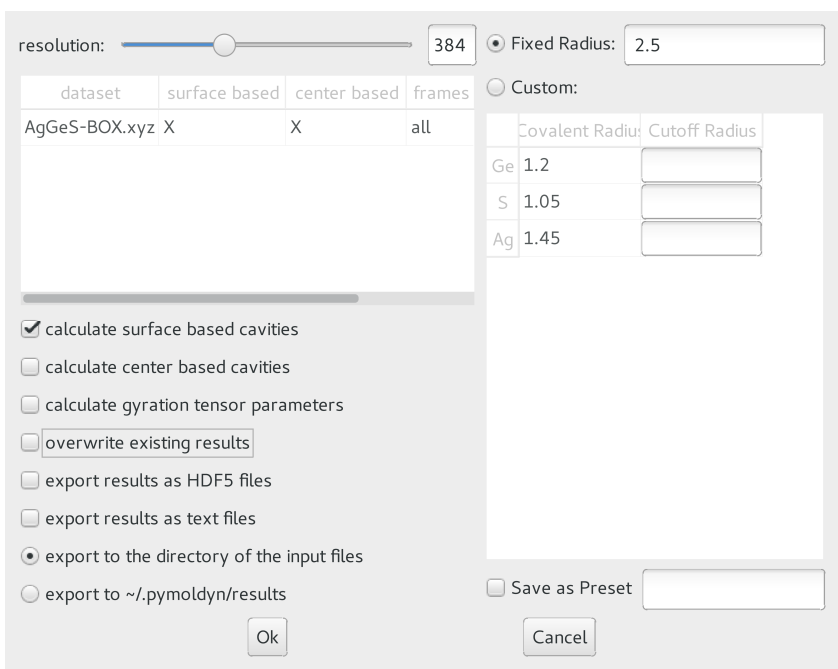

Fig. 3: Window prior to setting parameters for calculation.

The resolution and other quantities can then be changed as needed in the appropriate box, after which "OK" starts the calculation and leads to the screen shown in Fig. 4.

The program allows the generation of high-resolution images for publications and presentations, as well as creating videos that illustrate changes in structure (and cavities) as a function of time. Statistics generated by the program include surface areas and volumes (and the surface/volume ratio) of all cavities and domains, pair distribution functions and partial PDF, the distributions of bond types and of bond and dihedral angles, as well as the shape parameters discussed above. Pair distribution functions can be calculated and represented using seven window functions, and properties of individual atoms and cavities may be filtered. This information is available graphically, as an ASCII file, or as hdf5 output. For more details, see https://pgi-jcns.fzjuelich.de/portal/pages/pymoldyn-gui.html

A batch (command line interface) version is useful for generating multiple frames needed for videos and can be called via pymoldyn --batch <filename>

Further information concerning the batch version is provided in https://pgi-jens.fz-juelich.de/portal/pages/pymoldyn-cli.html

\section{Concluding Remarks}

The open source program pyMolDyn identifies cavities (vacancies, voids) in periodic systems of atoms in a unit cell with one of the seven 3D Bravais lattices. The program makes no assumptions about cavity shapes, allows for atoms of different sizes, and it calculates cavities defined in three ways: (a) "domains" determined by excluding spherical regions around each atom, (b) "centerbased" cavities determined by Dirichlet-Voronoi constructions for atoms and cavity centers, and (c) Dirichlet-Voronoi constructions for atoms and points of domain surfaces ("surface-based" cavities). The "split and merge" and "marching cubes" algorithms are utilized. The program is based on the GR3 and GR framework software [HRH15] and the input files use the . xyz format used in $\mathrm{Jmol} / \mathrm{JSmol}$ and other packages.

The size of systems that can be calculated depends on the number of atoms, the necessary resolution, and on the computing 


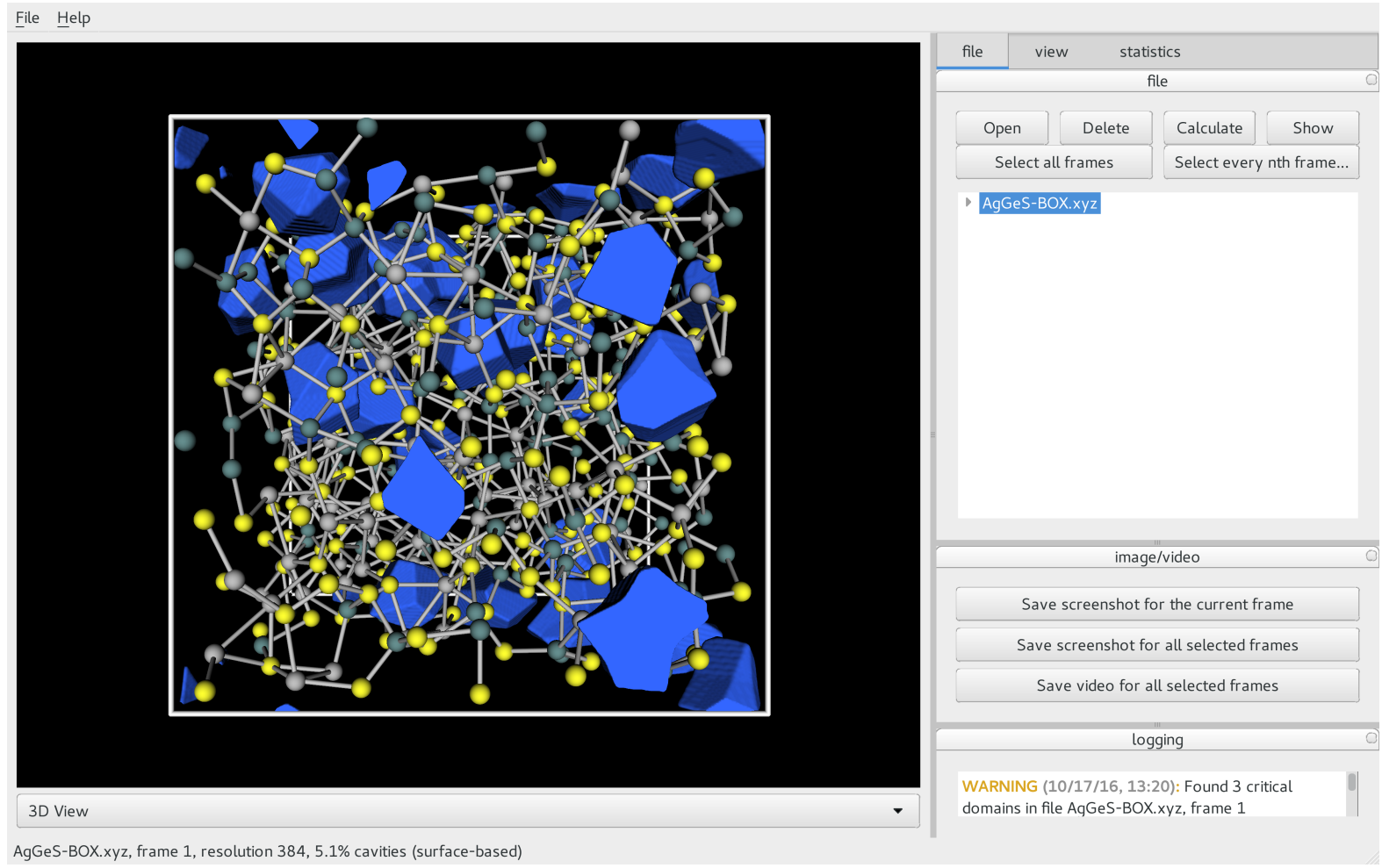

Fig. 4: Visualization of structure of Ag/Ge/S (silver: Ag, green: Ge, yellow: S) and surface-based cavities (blue).

\begin{tabular}{ll}
\hline resolution & time \\
\hline 128 & $4.0 \mathrm{~s}$ \\
192 & $6.5 \mathrm{~s}$ \\
256 & $15.3 \mathrm{~s}$ \\
384 & $43.0 \mathrm{~s}$ \\
512 & $158.3 \mathrm{~s}$ \\
\hline
\end{tabular}

TABLE 1: Time consumption for calculating surface and center based cavities for AgGeS-BOX. Xyz on a $2.5 \mathrm{GHz}$ Core $i 7$ with $16 \mathrm{~GB}$ RAM.

hardware (processor, memory) used. Systems with 500 atoms (for example AgGeS-BOX.xyz) can be computed in minutes on a 2.5 GHz Intel Core i7 (see TABLE 1).

Up to a resolution of 512 points the memory consumption is modest (4 GB of RAM are adequate). For higher resolutions over 10 GB of memory should be available.

Extensions to simplify calculations for isolated molecules and to allow the easy use of many-core, large memory computers are being implemented. We welcome suggestions and contributions to this ongoing project. Full details are available on https://pgijcns.fz-juelich.de/portal/pages/pymoldyn-main.html.

\section{Acknowledgments}

We thank numerous colleagues, particularly J. Akola, for suggestions and contributions. The program was developed to analyze the results of simulations of phase change materials carried out on supercomputers in the Forschungszentrum Jülich. We are grateful for computer time provided for this purpose by the JARA-HPC Vergabegremium on the JARA-HPC partition of JUQUEEN and for time granted on JUROPA and JURECA at the Jülich Supercomputer Centre.

\section{REFERENCES}

[AJ07] Akola, J. and Jones, R. O., Phys. Rev. B 2007, 76, 235201.

[AJ08] Akola, J. and Jones, R. O., J. Phys.: Condens. Matter 2008, 20, 465103.

[AJ12] Akola, J. and Jones, R. O., Phys. Status Solidi B 2012, 249, 1851-1860.

[LE11b] Lee, T. H. and Elliott, S. R., Phys. Rev. B 2011, 84, 094124.

[Hetal17] I. Heimbach, F. Rhiem, F. Beule, D. Knodt, J. Heinen and R. O. Jones, J. Comput. Chem. 2017, 38, 389-394.

[Be64] Bernal, J. D., Proc. R. Soc. A 1964, 280, 299-322.

[Fi70] Finney, J. L., Proc. R. Soc A 1970, 319, 479-493.

[Di50] Lejeune Dirichlet, G., J. Reine Angew. Mathematik 1850, 40, 209-227.

[Vo08] Voronoï, G., J. Reine Angew. Mathematik 1908, 134, 198-287.

[CL85] Chaki, T. K. and Li, J. C. M., Philos. Mag. B 1985, 51, 557-565.

[LAC13] Laghaei, R., Asher, S. A. and Coalson, R. D., J. Phys. Chem. B 2013, 117, 5271-5279.

[Vetal11] Voloshin, V. P., Medvedev, N. N., Andrews, M. N., Burri, R. R., Winter, R. and Geiger, A., J. Phys. Chem. B 2011, 115, 14217-14228.

[De34] Delaunay, B., Bulletin de l'Académie des Sciences de l'URSS. Classe des sciences mathématiques et naturelles 1934, 6, 793-800.

[AMS92] Arizzi, S., Mott, P. H. and Suter, U. W., J. Polym. Sci., Part B: Polym. Phys. 1992, 30, 415-426.

[VBM15] Voyiatzis, E., Böhm, M. C. and Müller-Plathe, F., Comput. Phys. Commun. 2015, 196, 247-254.

[LMNS00] Luchnikov, V. A., Medvedev, N. N., Naberukhin, Y. I. and Schober, H. R., Phys. Rev. B 2000, 62, 3181-3189.

[Ry09] Rycroft, C., Voro++: a three-dimensional Voronoi cell library in C++, United States Department of Energy, 2009. http://www.osti. gov/scitech/servlets/purl/946741

[MVLG06] Medvedev, N. N., Voloshin, V. P., Luchnikov, V. A. and Gavrilova, M. L., J. Comput. Chem. 2006, 27, 1676-1692.

[VNP] VNP program: Calculation of the Voronoi $S$-network (Additively weighted Voronoi Diagram), http://www.kinetics.nsc.ru/mds/ ?Software:VNP 
[LN88] Laakkonen, J. and Nieminen, R. M., J. Phys. C: Solid St. Phys. 1988, 21, 3663-3685.

[HP76] Horowitz, S. L. and Pavlidis, T., J. ACM 1976, 23, 368-388.

[LC87] Lorensen, W. E. and Cline, H. E., SIGGRAPH Comput. Graph. 1987, 21, 163-169.

[NY06] Newman, T. S. and Yi, H., Computers \& Graphics 2006, 30, 854-879.

[CBP10] Caravati, S., Bernasconi, M. and Parrinello, M., Phys. Rev. B 2010, $81,014201$.

[KAJ14] Kalikka, J., Akola, J. and Jones, R. O., Phys. Rev. B 2014, 90, 184109.

[Metal11] Matsunaga, T., Akola, J., Kohara, S., Honma, T., Kobayashi, K., Ikenaga, E., Jones, R. O., Yamada, N., Takata, M. and Kojima, R., Nature Mater. 2011, 10, 129-134.

[Ep69] Epanechnikov, V. A., Theory Probabl. Appl. 1969, 14, 153-158.

[TS85] Theodorou, D. N. and Suter, U. W., Macromolecules (Washington, DC, U.S.), 1985, 18, 1206-1214.

[HRH15] Heinen, J., Rhiem, F., Felder, C., Beule, F., Brandl, G., Dück, M., Goblet, M., Heimbach, I., Kaiser, D., Klinkhammer, P., Knodt, D., Nesselrath, R., Westphal, E. and Winkler, J., GR-a universal framework for visualization applications, 2015. http://grframework.org

[OB] Open babel file: element.txt, http://sourceforge.net/p/openbabel/ code/5041/tree/openbabel/trunk/data/element.txt

[Cetal08] Cordero, B., Gomez, V., Platero-Prats, A. E., Reves, M., Echeverria, J., Cremades, E., Barragan, F. and Alvarez, S., Dalton Trans. 2008, 2832-2838.

[Jmol] Jmol: an open-source Java viewer for chemical structures in 3D, http://www.jmol.org or http://wiki.jmol.org/. JSmol is an implementation of Jmol that does not require Java and runs on any web browser supporting HTML5.

[SF] Jmol colors, http://jmol.sourceforge.net/jscolors/

[Aetal15] Akola, J., Beuneu, B., Jones, R. O., Jóvári, P., Kaban, I., Kolář, J., Voleská, I. and Wágner, T., J. Phys.: Condens. Matter 2015, 27, 485304. 